\title{
Look \& Listen: Sonification and Visualization of Multiparameter Micrographs
}

\author{
Tim W. Nattkemper*, Thomas Hermann ${ }^{\dagger}$, Walter Schubert ${ }^{\ddagger \S}$, Helge Ritter ${ }^{\dagger}$ \\ *Applied Neuroinformatics Group, Faculty of Technology \\ Bielefeld University, PO Box 100131, D-33501 Bielefeld, Germany \\ Email: tnattkem@techfak.uni-bielefeld.de \\ $\ddagger$ Meltec Ltd., Magdeburg, Germany \\ $\S$ Institute of Medical Neurobiology, University of Magdeburg, Germany \\ ${ }^{\dagger}$ Neuroinformatics Group, Faculty of Technology, Bielefeld University, Germany
}

\begin{abstract}
Multiparameter imaging techniques provide large numbers of high-dimensional image data in modern biomedical research. Besides algorithms for image registration, normalization and segmentation, new methods for interactive data exploration must be proposed and evaluated. We propose a new approach for auditory data representation, based on sonification. The approach is applied to a multiparameter image data set, generated with immunofluorescence techniques and compared to a conventional visualization approach and to a combination of both. For comparison, a psychophysical experiment was conducted, in which one standard evaluation procedure is modeled. Our results show, that all three approaches lead to comparable evaluation accuracies for all subjects. We conclude, that both, acoustical and visual approaches can be combined to display data sets of large dimensionality.
\end{abstract}

Keywords-Biomedical Imaging, Multiparameter Image Analysis, Exploratory Data Analysis, Visualization, Sonification

\section{INTRODUCTION}

In recent years, substantial progress has been achieved in the development and application of optical microscopy in molecular and cellular biology. Advanced staining techniques and multiband imaging allow highly sensitive visualization of macromolecules and compartments in the cells (e. g. [1], [2]). In multimodal imaging approaches, different imaging techniques are merged to give additional insight into cell functions [3]. In this paper we summarize all these approaches as multiparameter imaging approaches. The recorded data is a stack of $n$ parameter images of the same site in the sample. In general, these multiparameter imaging approaches are applied to cell samples from different specimens or in certain stress conditions to get more comprehensive descriptions of spatial and temporal molecular dynamics in the cell [4]. The basic aspect of such multiparameter approaches in biomedicine is that the information about spatial correlations between the mapped channels is preserved, which is a valuable feature not owned by many other methods, as e. g. gel-electrophoresis or microarrays.

After post-processing and registration, the data usually has to be analyzed by $(a)$ direct manual exploration (generally by visual inspection) and/or (b) statistical datamining methods. To perform $(b)$, quantitative data has to be extracted from the images. To this end, it is inevitable to perform an image segmentation interactively or full automatically. This is often impractical, because $(i)$ the manual evaluation is error-prone (especially on large datasets) and (ii) for an automatic evaluation with image processing algorithms a constant quality in the data is required, resulting in lots of time consuming standardization work. In this work, therefore we focus on the evaluation of methods to support $(a)$, the direct manual exploration of multiparameter data. Manual exploration in multiparameter images aim at the local discovery of molecular patterns and their association with cellular functions. These observations shall culminate in the development of integrated models of cell functions. Simulation and graphical display of such models in virtual cells are corner stones in the rapid evolving field of systems biology [5]. Because a comprehensive and efficient analysis of multiparameter images is vital to this field, innovative methods for the manual exploration of this data are urgently required. In this paper we compare a traditional visualization and a new sonification approach to display multiparameter image data. For first time, we apply visualization, sonification and the combination of both to the field of exploratory multiparameter image analysis and quantitatively evaluate the approach in an experiment.

The task of manual exploration of multiparameter image exploration can be described as follows: In a single twodimensional referential display of the sample, a region-ofinterest (ROI) is selected using a pointing device (e. g. a cursor controlled by a computer mouse) and the image parameters of the object within the ROI are displayed ${ }^{1}$. The parameter display must be designed to meet requirements that follow from the general research framework outlined above and from individual research tasks in single laboratories. Any display must meet the following terms, to be applicable in the general framework of multiparameter image analysis:

Identity: Objects with identical biological parameters must be identically displayed. To this end, a normalization and

${ }^{1}$ To analyze the entire content of a multiparameter image, the parameters of all ROIs can in principle also be visualized at once. But this must be preceeded by a full segmentation of the image. Because we focus on direct manual exploration without time-consuming comprehensive segmentation we display the parameters of only one selected region at a time. 


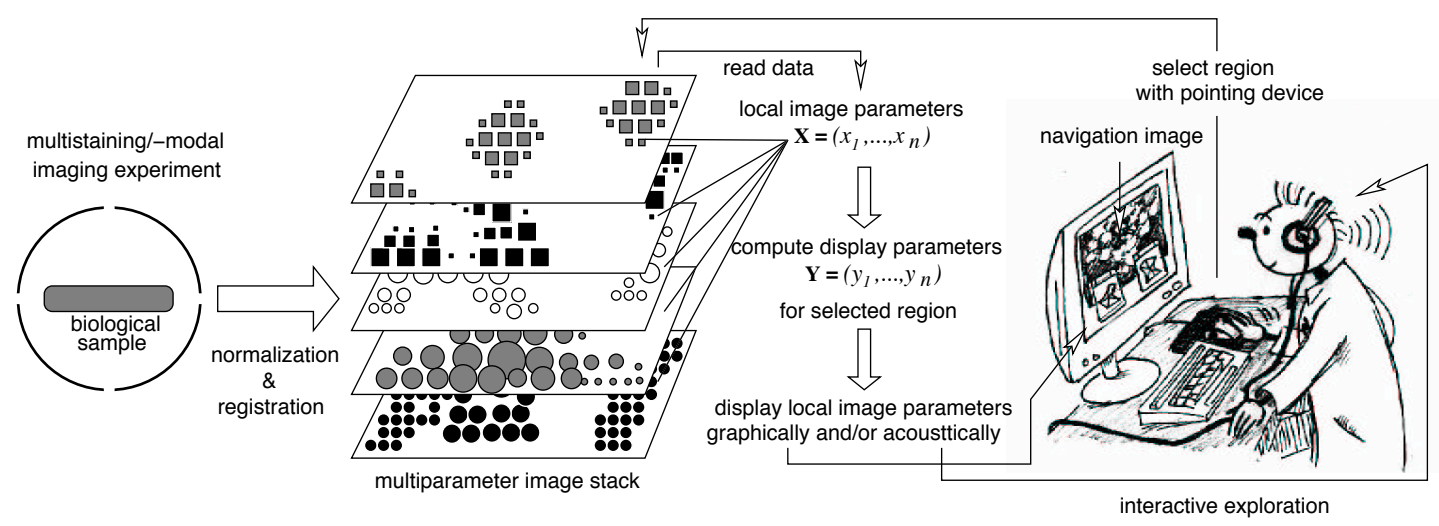

Fig. 1. The set up of interactive exploration of multiparameter images with visualization and sonification methods

thresholding procedure is applied to the signal values.

Similarity: Displays for biological parameters of two regions must be perceived similar or different, corresponding to their distance in the parameter space. The distance in the $n$ dimensional parameter space can be measured using a metric function (e. g. an Euclidean distance).

Extensibility: The display technique should not be restricted to $n$-dimensional patterns and in principle has to be extensible to additional parameters.

Stability: The display should not change drastically in case of missing values. Missing values can occur at the imaging or the registration step.

Compactness: Display and visual inspection should not take too much time in order to enable exploration in appropriate time. It also has to allow the display of parameters of several selected regions.

\section{EXPLORATORY MICROGRAPH ANALYSIS}

Basis of our study is as an exemplary data set of multichannel fluorescence images which has been selected from the same set as the data in [6]. The stack shows a set of cells in a blood sample. In each image of the stack, the intensity values identify the presence of a molecule via immunofluorescence [7]. In principle, many more protein signals than illustrated here can be captured in one biological sample by using new robotic imaging approaches.

\section{A. Visualization approach}

Most works about visual exploration of biomedical data propose and discuss approaches to provide a graphical display for revealing a multivariate structure, in particular similarities and clusters [8]. In this context graphical primitives like autoglyphs or icon plots are used to support a scatter plot or other visualizations of equivalent type and are preceded by the extraction of numerical data from the image. Usually, the parameter vectors are visualized independently upon their location. But the spatial information is an essential feature of the microscopy approach, that enables the researcher to identify the molecules at the anatomical site in contrast to other experimental approaches like e. g. 2D electrophoresis and microarrays. In other works stick figures are used to compute
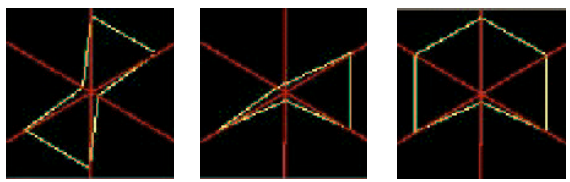

Fig. 2. Three example star glyphs of three different six dimensional parameter vectors are shown.

a texture map from the $n$-dimensional image stack. The local texture in the map is determined by the local image parameter values that control the shape of the texton (or texel) which is a stick figure. The technique is limited to reveal raw image structures. A more promising approach is the computation of so-called Chernoff faces [9], [10] to represent the parameters of image regions. Each parameter value controls a single image feature in a graphically rendered image. Thus, for different parameters faces of different emotional expression are computed. One advantage of this method is that missing values are represented by missing features in the face, but the rest of the face obeys the similarity term, stated above. Nevertheless in the context of this work, the Chernoff faces are not further considered because it makes no sense to associate an emotional state to a molecular expression pattern, which is a priori neutral.

In this work we display the local image parameters with so called star glyphs [11] which belong to the family of sequential icon plots. Glyphs are well suited to reveal correlations in the data, which is one of the key issues in data analysis. Additionally, glyphs can be positioned at their original location in the image, which is vital to find regularities in the relative positions of molecular patterns. To display the parameters of one region, it is represented by a star or a polygon. The length of the vector to each vertex corresponds to the thresholded value of a particular variable (see Fig. 2).

\section{B. Sonification approach}

Sonification is the presentation of data using sound [12]. As in visualization techniques, the data is transformed into a modality in which humans have extraordinarily highdeveloped skills in discerning and comparing patterns. The auditory presentation of high-dimensional patterns is a very promising strategy, since we usually are able to process a 


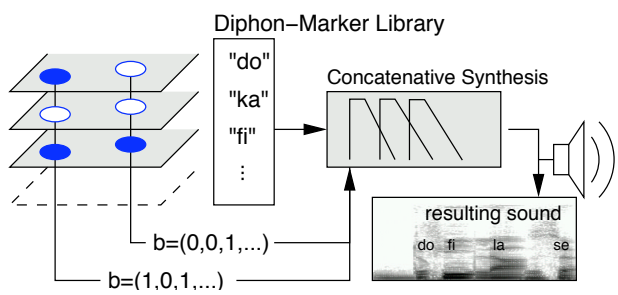

Fig. 3. Illustration of concatenative synthesis of cell pattern sonifications, using recorded diphon sound samples.

multitude of sound attributes at the same time, e.g. we are able to perceive the pitch, loudness, roughness, brightness, localization, duration, reverberation, etc. of a piano tone in a single moment. This mapping from data to sound parameters (called Parameter Mapping) however suffers from various problems, for instance nonlinear scales of acoustic parameters or lack of orthogonality between the attributes. Some of them can be circumvented by using more complex sound segments to represent a single high-dimensional vector. Different strategies for this have been proposed in [6], using rhythmical, harmonic -and by combining them- melodic musical motives for representing a single multi-dimensional vector as for instance the vector of image intensities through the image stack at a certain location. The different aspects reported above like extensibility etc., can be easily incorporated into the structure of the sound patterns, although the medium sound poses here some principal limitations, e.g. using a chord of tones, it becomes difficult to discern more than about 8 tones at the same time. Also, musically untrained listeners have disadvantages in processing and comparing the stimuli. This led us to employing sounds from a domain where most listeners have high-developed perceptual skills without the need of additional training: two promising domains for this are environmental sounds and linguistic sounds. The latter were finally employed to render "artificial words" for presenting a data vector. Practically this is done by using a concatenative synthesis of diphone sequences like 'do', 'ka', 'fi', 're', etc. This sonification strategy is illustrated in Figure 3. Thus, a diphon becomes the acoustic marker for representing a biomedical protein marker. The pattern $(0,1,0,0,1,0,1)$ is thus represented by the spoken word 'ka-sela' whereas $(0,0,1,0,1,0,1)$ would become 'fi-se-la'. As a result, similar patterns are easily recognizable from similar word forms. Also, since we are highly trained to process similar stimuli in real speech sounds, the pattern can be processed within a rather short time of about one second. This technique is applied here in its simplest form, leaving the huge range of lingual variations in prosody, like pitch, rhythm, accentuation, voice timbre unused, so that this way of mapping data to acoustic stimuli still has great potential for further extensions.

\section{Combination approach}

In a third approach, the region parameters are displayed in both ways simultaneously to study the effect of redundant display on the evaluation performance.

\section{EXPERIMENTAL SETUP}

One basic task in the analysis of multiparameter images is exploration of images for regularities in the data. One basic kind of regularity is a "parameter pattern" in the data which is observed at several regions in the image (or in different images from different samples). To analyze the efficiency and accuracy of the display approaches introduced above, the detection of such repeated "parameter patterns" task is modeled in an experiment, in which the subjects had to solve this task in referential data sets. In one experimental run the subject had to detect and mark regions of identical parameters in a sixdimensional multiparameter image stack using one of the three display techniques. The regions are cell bodies in a blood sample. Because the subjects were not biological experts the regions (i. e. cell bodies) were highlighted in order to guide their search in a reasonable way. One cell was highlighted differently to be the reference cell for the identity search.

Each subject had to perform ten runs. The performance of the subjects was used to assess the efficiency of visual, auditory and combination display. The efficiency was measured from two experimental outcomes: duration of the entire evaluation and evaluation accuracy. The experiment was performed by 60 non-expert subjects, divided into three groups of same size, called V, A, and C. After indicating the start of the experimental session by pressing the start button, the subject searched the image for cells which were identical to the reference cell. To judge the similarity of the inspected and the reference cell, a left mouse button press offers a (visual, auditory, or combined) display of the inspected cell's parameter pattern. If a cell's parameters were perceived as being different to the reference cell, the cell was marked with the middle mouse button and a red cross was displayed on the cell in the image. In case of a cell identical to the reference cell the subject marked it with a green cross by clicking with the right mouse button on the cell. The subject could convert marks by pointing and marking the cell again. The session was finished by activating a stop button. For each session, the marks and the session duration were recorded. Two kinds of errors were evaluated: cells wrongly labeled as being identical to the reference cell (false positives) or wrongly marked as being different (false negatives). We followed the simplest possible hypothesis and assumed that both kinds of errors happen to all subjects with the same probability.

\section{RESUlts}

The first interesting factor is the evaluation accuracy, i. e. the number of errors in one experimental run. Two subjects in group $\mathrm{V}$ showed an outstanding high error rate, caused by individual handling problems. Thus, they are classified as outliers, so they are not considered in the statistical analysis. For each subject the number of wrong marked cells $x_{i}^{(k)}$ with $i=1, \ldots, n^{(k)}\left(n^{(k)}=18\right.$ for the $\mathrm{V}$ group and $n^{(k)}=20$ for group $\mathrm{A}$ and $\mathrm{C}$ ), and $k \in\{V, A, C\}$ is determined and for each approach mean and variance $\left(\mu_{x}^{(k)}, \sigma_{x}^{(k)}\right)$ of the error rate is computed (see Tab. I). A F-test is applied to 


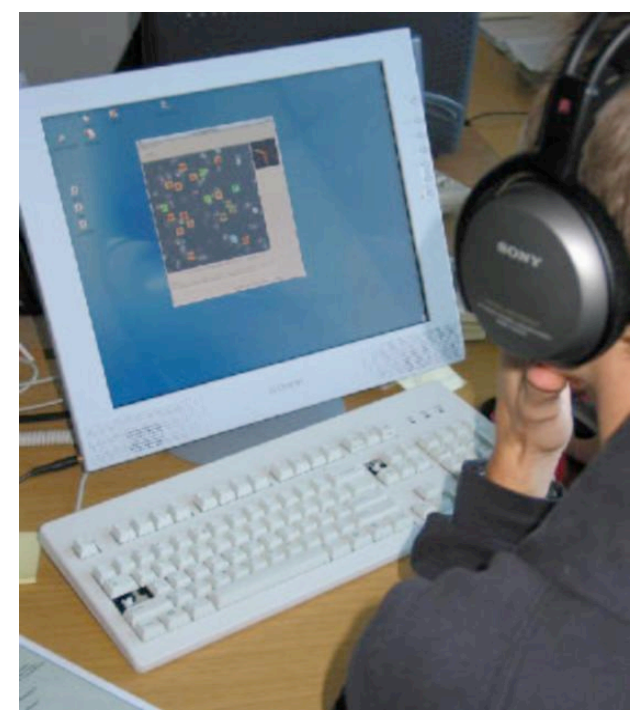

Fig. 4. Experimental set-up of visualization/sonification-based exploration

TABLE I

ERROR RATES AND EVALUATION DURATIONS FOR VISUALIZATION, SONIFICATION OR COMBINATION DISPLAY.

\begin{tabular}{|c|c|c|c|c|c|c|c|}
\hline \multicolumn{3}{|c|}{ Error rates in subject groups } & \multicolumn{4}{|c|}{ Evaluation duration in subject groups } \\
\hline$k=$ & $V$ & $A$ & $C$ & $k=$ & $V$ & $A$ & $C$ \\
\hline$n^{(k)}$ & 18 & 20 & 20 & $n^{(k)}$ & 165 & 178 & 177 \\
$\mu_{x}^{(k)}$ & 1.28 & 1.45 & 1.75 & $\mu_{t}^{(k)}$ & 49.86 & 60.17 & 55.88 \\
$\sigma_{x}^{(k)}$ & 1.99 & 2.11 & 2.65 & $\sigma_{t}^{(k)}$ & 15.38 & 18.12 & 17.33 \\
\hline
\end{tabular}

the data to compare the variances and showed no change in variance between $\mathrm{V}, \mathrm{A}$ and $\mathrm{C}$ experiments. Based on the $\mathrm{F}$ test results, a T-test is applied to the data and showed that no significant change in the error rate can be observed between different display modalities. The second interesting factor is the evaluation time. For all experimental runs, the evaluation time $t_{i j}^{(k)}$ with $i=1, \ldots, 20$ (subject ID), $j=1, \ldots, 10$ (run ID) and $k \in\{V, A, C\}$ was recorded. To compare the three approaches, again the means and variance $\left(\mu_{t}^{(k)}, \sigma_{t}^{(k)}\right)$ were computed. To eliminate any dependencies with the factor error rate, only those experimental runs were considered that contained no errors, i. e. the subjects marked all cells correctly. The means and standard deviations are shown in Tab. I.

A F-test showed the absence of any significant differences between the variances for the three approaches with a significance level of $95 \%$ concerning the evaluation duration, apart from a comarison of $\mathrm{V}$ with $\mathrm{A}$. Based on these findings a $\mathrm{T}$ test was applied and showed that the subjects from group V required the shortest time, the subjects from the sonification group performed in the slowest time both on a significance level of $95 \%$. However, the overall difference in evaluation time is less than $15 \%$.

\section{DISCUSSION}

We proposed a new approach for the display of cellular parameters in multiparameter images. The approaches are tested and evaluated in a psychophysical experiment. The experiment modeled one basic type of task in the evaluation of multiparameter images.

The experimental data shows, that with regard to error rate visualization and sonification approaches are equally suited to support the analysis of cellular parameters. Thus, they can be combined in a equitable fashion. For example, one set of parameters can be displayed graphically and the other set can be displayed acoustically.

Another interesting observation is, that for the chosen experimental setting the evaluation time of single visualization alone is the shortest for all three display techniques. This could be caused by the low number of six parameters. The star glyphs of different patterns can be well discriminated for a low number of vectors, which can not be expected for a larger number of parameters. Thus, in future experiments the number of parameters will be increased to study the dependence of evaluation duration on the parameter number.

We observed that the combination approach did not improve the performance. This is surprising, because in the literature an increase in performance was reported in comparable studies [13]. Again, this will be further studied for larger numbers of parameters.

\section{ACKNOWLEDGMENTS}

This work was supported by the BMBF grant BMBF0312844. Special thanks go to T. Bovermann, B. Hafer, C. Lange, O. Lichte, W. Timm, J. Vompras for computational and experimental support.

\section{REFERENCES}

[1] M. V. Boland, "Automated classification of cellular protein localization patterns obtained via fluorescence microscopy," in International Conference of the IEEE Engineering in Medicine and Biology Society, 1997.

[2] M. P. Oksvold, E. Skarpen, J. Widerberg, and H. S. Huitfeldt, "Fluorescent histochemical techniques for analysis of intracellular signaling," Journal of Histochemistry \& Cytochemistry, 2002.

[3] R. A. Wind, "An integrated confocal and magnetic resonance microscope for cellular research." Journal of Magnetic Resonance, vol. 147, 2000.

[4] D. L. Taylor, E. S. Woo, and K. A. Giuliano, "Real-time molecular and cellular analysis: the new frontier of drug discovery." Current Opinion Biotech., vol. 12, pp. 75-81, 2001.

[5] H. Kitano, "Perspective in systems biology," New Generation Computing, vol. 18, pp. 199-216, 2000.

[6] T. Hermann, T. Nattkemper, W. Schubert, and H. Ritter, "Sonification of multi-channel image data," in Proc. of the Mathematical and Engineering Techniques in Medical and Biological Sciences (METMBS 2000) METMBS, 2000, pp. 745-750.

[7] W. Schubert, Topological Proteomics, Toponomics, MELK-Technology. Berlin-Heidelberg-New York: Springer Verlag, 2003, ch. Proteomics of Microorganismus: Fundamental Aspects and Application, Advances in Biochemical Engineering/Biotechnology, in press.

[8] A. Buja, D. Cook, and D. Swayne, "Interactive high-dimenional data visualization," Journal of Computational and Graphical Statistics, vol. 5, no. 1, pp. 78-99, 1996.

[9] H. Chernoff, "The use of faces to represent points in n-dimensional pace graphically," Department of Statistics, Stanford University, Tech. Rep. RN NR-042-993, 1971.

[10] M. Smith, R. Taffler, and L. White, "Cartoon graphics in the communication of accounting information for management decision making," Journal of Applied Management Accounting Research, vol. 1, no. 1, pp. 31-50, 2002.

[11] M. O. Ward and A. R. Martin, "High dimensional brushing for interactive exploration of multivariate data," in Proc. of IEEE Visualization, 1995, pp. 271-278.

[12] G. Kramer, Ed., Auditory Display - Sonification, Audification, and Auditory Interfaces. Addison-Wesley, 1994. 
[13] M. Williams, S. Smith, and G. Pecelli, "Computer-human interface issues in the design of an intelligent workstation for scientific visualization," SIGCHI Bulletin, vol. 21, no. 4, pp. 44-49, 1990. 